\title{
POSITIVELY CURVED KAEHLER SUBMANIFOLDS
}

\author{
ANTONIO ROS
}

\begin{abstract}
In this note we prove that if the holomorphic curvature of a compact Kaehler submanifold in the complex projective space is bigger than $\frac{1}{2}$, then it is totally geodesic.
\end{abstract}

Introduction. Let $\mathrm{CP}^{m}$ be the $m$-dimensional complex projective space with the Fubini-Study metric of constant holomorphic sectional curvature $c=1$. We will prove the following result.

THEOREM. Let $M^{n}$ be a compact Kaehler submanifold of complex dimension $n$ immersed in $C P^{m}$. If every holomorphic sectional curvature of $M^{n}$ is greater than $\frac{1}{2}$, then $M^{n}$ is totally geodesic in $C P^{m}$.

The above Theorem was conjectured by K. Ogiue [1]. Some partial solutions are collected in [1]. As the Frankel conjecture is true [2], we remark that under the hypothesis of the theorem, $M^{n}$ is biholomorphic to $\mathrm{CP}^{n}$. We use the notation of [1].

1. Preliminaries. Let $M^{n}$ be a Kaehler submanifold of complex dimension $n$ in the complex projective space $\mathrm{CP}^{m}$. We denote by $J$ and $g$ the complex structure and the metric of constant holomorphic sectional curvature $c=1$ on $\mathrm{CP}^{m}$. Let $\bar{\nabla}$ and $\nabla$ be the Riemannian connection of $\mathrm{CP}^{m}$ and $M^{n}$, respectively, $\sigma$ the second fundamental form of the immersion, $A$ and $\nabla^{\perp}$ the Weingarten endomorphism and the normal connection of $M^{n}$ in $\mathrm{CP}^{m}$, and $\nabla \sigma$ the covariant derivative of $\sigma$.

We define the second covariant derivative of $\sigma$ by

$$
\begin{array}{r}
\left(\nabla^{2} \sigma\right)(X, Y, Z, W)=\nabla_{X}^{\perp}((\nabla \sigma)(Y, Z, W))-(\nabla \sigma)\left(\nabla_{X} Y, Z, W\right) \\
-(\nabla \sigma)\left(Y, \nabla_{X} Z, W\right)-(\nabla \sigma)\left(Y, Z, \nabla_{X} W\right)
\end{array}
$$

for any vector fields $X, Y, Z, W$ tangent to $M^{n}$.

Let $\bar{R}, R$ and $R^{\perp}$ denote the curvature tensors associated with $\bar{\nabla}, \nabla$ and $\nabla^{\perp}$, respectively. Then we have

$$
\begin{gathered}
\bar{R}(\bar{X}, \bar{Y}) \bar{Z}=\frac{1}{4}\{g(\bar{Y}, \bar{Z}) \bar{X}-g(\bar{X}, \bar{Z}) \bar{Y}+g(J \bar{Y}, \bar{Z}) J \bar{X} \\
-g(J \bar{X}, \bar{Z}) J \bar{Y}+2 g(\bar{X}, J \bar{Y}) J \bar{Z}\} \\
R(X, Y) Z=\bar{R}(X, Y) Z+A_{\sigma(Y, Z)} X-A_{\sigma(X, Z)} Y \\
R^{\perp}(X, Y, \xi, \eta)=\bar{R}(X, Y, \xi, \eta)+g\left(\left[A_{\xi}, A_{\eta}\right] X, Y\right)
\end{gathered}
$$

Received by the editors January 26, 1984.

1980 Mathematics Subject Classification. Primary 53C40; Secondary 53C55. 
for vector fields $\bar{X}, \bar{Y}, \bar{Z}$ tangent to $\mathrm{CP}^{m}, X, Y, Z$ tangent to $M^{n}$ and $\xi, \eta$ normal to $M^{n}$.

Moreover, $\sigma$ and $\nabla \sigma$ are symmetric, and

$$
\begin{aligned}
\left(\nabla^{2} \sigma\right)(X, Y, Z, W)-\left(\nabla^{2} \sigma\right)(Y, X, Z, W)=R^{\perp}(X, Y) \sigma(Z, W) \\
-\sigma(R(X, Y) Z, W)-\sigma(Z, R(X, Y) W) .
\end{aligned}
$$

We also consider the relations

$$
\begin{gathered}
\sigma(J X, Y)=\sigma(X, J Y)=J \sigma(X, Y), \\
A_{J \xi}=J A_{\xi}, \quad J A_{\xi}=-A_{\xi} J, \quad \nabla_{X}^{\perp} J \xi=J \nabla_{X}^{\perp} \xi, \\
(\nabla \sigma)(J X, Y, Z)=(\nabla \sigma)(X, J Y, Z) \\
=(\nabla \sigma)(X, Y, J Z)=J(\nabla \sigma)(X, Y, Z) .
\end{gathered}
$$

Finally if $u$ is a unit tangent vector to $M^{n}$, then the holomorphic sectional curvature $H$ of $M^{n}$ determined by $u$ is given by

$$
H(u)=1-2\|\sigma(u, u)\|^{2} .
$$

2. Proof of Theorem. Let $\Pi: U M \rightarrow M$ and $U M_{p}$ be the unit tangent bundle of $M$ and its fiber over $p \in M$, respectively. We define a function $f: U M \rightarrow \mathbf{R}$ by $f(u)=\|\sigma(u, u)\|^{2}$ for any $u$ in $U M$. From (1.9), the hypothesis $H>\frac{1}{2}$ is equivalent to $f<\frac{1}{4}$.

Fix $v$ in $U M_{p}, p \in M$. For any $u$ in $U M_{p}$, let $\gamma_{u}(t)$ be the geodesic in $M$ given by the initial conditions $\gamma_{u}(0)=p, \gamma_{u}^{\prime}(0)=u$. By parallel translating $v$ along $\gamma_{u}(t)$, we obtain a vector field $V_{u}(t)$. Put $f_{u}(t)=f\left(V_{u}(t)\right)$. By direct computation we obtain

$$
\frac{d}{d t} f_{u}(t)=2 g\left((\nabla \sigma)\left(\gamma_{u}^{\prime}, V_{u}, V_{u}\right), \sigma\left(V_{u}, V_{u}\right)\right)(t)
$$

and

$$
\frac{d^{2}}{d t^{2}} f_{u}(0)=2 g\left(\left(\nabla^{2} \sigma\right)(u, u, v, v), \sigma(v, v)\right)+2\|(\nabla \sigma)(u, v, v)\|^{2} .
$$

As $\left(\nabla^{2} \sigma\right)(J v, J v, v, v)=\left(\nabla^{2} \sigma\right)(J v, v, J v, v)$, from (1.5) we have

$$
\begin{aligned}
g\left(\left(\nabla^{2} \sigma\right)\right. & \left.(J v, J v, v, v)-\left(\nabla^{2} \sigma\right)(v, J v, J v, v), \sigma(v, v)\right) \\
= & R^{\perp}(J v, v, \sigma(J v, v), \sigma(v, v)) \\
& -2 g(\sigma(R(J v, v) v, J v), \sigma(v, v)) .
\end{aligned}
$$

From (1.2)-(1.4), (1.6), (1.7) and (2.3), and taking into account that $\left(\nabla^{2} \sigma\right)(v, J v, J v, v)=-\left(\nabla^{2} \sigma\right)(v, v, v, v)$, we obtain

$$
\begin{aligned}
& g\left(\left(\nabla^{2} \sigma\right)(J v, J v, v, v), \sigma(v, v)\right) \\
&=-g\left(\left(\nabla^{2} \sigma\right)(v, v, v, v), \sigma(v, v)\right) \\
&+\frac{3}{2}\|\sigma(v, v)\|^{2}-6 g\left(\sigma(v, v), \sigma\left(A_{\sigma(v, v)} v, v\right)\right) .
\end{aligned}
$$


Hence from (1.8), (2.2) and (2.4) we have

$$
\begin{aligned}
\frac{d^{2}}{d t^{2}} f_{v}(0)+\frac{d^{2}}{d t^{2}} f_{J v}(0)= & 3\|\sigma(v, v)\|^{2}-12 g\left(\sigma(v, v), \sigma\left(A_{\sigma(v, v)} v, v\right)\right) \\
& +4\|(\nabla \sigma)(v, v, v)\|^{2} .
\end{aligned}
$$

$U M$ being compact, $f$ attains the maximum at some vector in $U M$. We suppose that this vector is $v$. Hence

$$
\frac{d^{2}}{d t^{2}} f_{v}(0)+\frac{d^{2}}{d t^{2}} f_{J v}(0) \leqslant 0
$$

For any $u$ in $U M_{p}$, with $g(v, u)=0$, let $\alpha(s)$ be a curve in the sphere $U M_{p}$ such that $\alpha(0)=v, \alpha^{\prime}(0)=u$. As $v$ is a critical point of $f$ we have

$$
\frac{d}{d s}(f \circ \alpha)(0)=4 g(\sigma(v, v), \sigma(v, u))=0,
$$

or equivalently $g\left(A_{\sigma(v, v)} v, u\right)=0$ for all $u \in U M_{p}$ with $g(v, u)=0$. Hence

$$
A_{\sigma(v, v)} v=\|\sigma(v, v)\|^{2} v \text {. }
$$

From (2.5) and (2.7) we obtain

$$
\frac{d^{2}}{d t^{2}} f_{v}(0)+\frac{d^{2}}{d t^{2}} f_{J v}(0)=3 f(v)(1-4 f(v))+4\|(\nabla \sigma)(v, v, v)\|^{2} .
$$

Finally, from (2.6) and (2.8) we conclude that $f(v)(1-4 f(v)) \leqslant 0$. From the hypothesis, $f(v)<\frac{1}{4}$. Hence $f(v)=0, M^{n}$ is totally geodesic, and we have proved the Theorem.

\section{REFERENCES}

1. K. Ogiue, Differential geometry of Kaehler submanifolds, Adv. in Math. 13 (1974), 73-114.

2. Y. T. Siu and S. T. Yau, Compact Kaehler manifolds of positive bisectional curvature, Invent. Math. 59 (1980), 189-204.

Departamento de Geometria, facultad de Ciencias, Universidad de Granada, Granada, SPAIN 